\title{
Differential dopamine release dynamics in the nucleus accumbens core and shell track distinct aspects of goal-directed behavior for sucrose
}

\author{
Fabio Cacciapagliaa ${ }^{a, b}$, Michael P. Saddoris ${ }^{b}$, R. Mark Wightman ${ }^{a, c}$, and Regina M. Carelli ${ }^{b, c,{ }^{*}}$ \\ aDepartment of Chemistry, University of North Carolina, Chapel Hill, NC 27599, USA \\ bDepartment of Psychology, University of North Carolina, Chapel Hill, NC 27599, USA \\ 'Neuroscience Center, University of North Carolina, Chapel Hill, NC 27599, USA
}

\section{Abstract}

\begin{abstract}
Mesolimbic dopamine projections to the nucleus accumbens (NAc) have been implicated in goaldirected behaviors for natural rewards and in learning processes involving cue-reward associations. The NAc has been traditionally subdivided into two anatomically distinct subregions with different functional properties: the shell and the core. The aim of the present study was to characterize rapid dopamine transmission across the two NAc sub-regions during cuesignaled operant behavior for a natural (sucrose) reward in rats. Using fast-scan cyclic voltammetry (FSCV) we observed differences in the magnitude and dynamics of dopamine release events between the shell and core. Specifically, although cue-evoked dopamine release was observed in both sub-regions, it was larger and longer lasting in the shell compared with the core. Further, secondary dopamine release events were observed following the lever press response for sucrose in the NAc shell, but not the core. These findings demonstrate that the NAc displays regional specificity in dopamine transmission patterns during cued operant behavior for natural reward.
\end{abstract}

\section{Keywords}

Behavior; Nucleus accumbens; Dopamine; Voltammetry; Electrochemistry; Sucrose

\section{Introduction}

The nucleus accumbens (NAc) is a key neural structure involved in processing information about reward and learning processes governing stimulus-reward associations. It is typically subdivided in two main sub-regions: the shell and core (Heimer et al., 1991; Zahm and Brog, 1992; Jongen-Relo et al., 1994) that differ in their anatomical connections and function. Although some differences are found in the density of cortical inputs (Corbit et al., 2001), the major distinctions are observed in their efferent projections. That is, the shell projects mainly to limbic regions (i.e., lateral hypothalamus, ventral part of the bed nucleus of the stria terminalis and ventral tegmental area), whereas core projections are directed primarily to motor-related structures (i.e., caudate putamen, globus pallidus and substantia nigra) (Zahm and Brog, 1992; Zahm and Heimer, 1993; Corbit et al., 2001). 
As such, the NAc shell and core display different functional properties in the expression of motivated behavior, in cue-reward learning, and in encoding the primary reinforcing properties of natural rewards. Microdialysis studies show that, in nondeprived rats, novel palatable food stimulates dopamine transmission preferentially in the NAc shell (Bassareo and Di Chiara, 1997), whereas food-conditioned stimuli induce increases in extracellular dopamine in the NAc core (Bassareo and Di Chiara, 1999). Inactivation of the NAc core (not shell) disrupts cue-induced reinstatement of both drug and food seeking behavior (Fuchs et al., 2004; Floresco et al., 2008). Further, stimulation of NAc dopamine transmission by natural and/or drug rewards and associated cues appear to occur in a reward-specific manner. For instance, cocaine predictive cues evoke increases in phasic dopamine release within the NAc core, but not the shell (Aragona et al., 2009), whereas morphine conditioned stimuli (CS) increase extracellular dopamine preferentially in the NAc shell (Bassareo et al., 2007).

Using fast-scan cyclic voltammetry (FSCV), we previously reported that cues signaling sucrose reward elicit increases in phasic dopamine release within the NAc core (Roitman et al., 2004; Day et al., 2007; Jones et al., 2010a; Cacciapaglia et al., 2011) and that lever press responses for sucrose reinforcement occur at the peak of dopamine surges (Roitman et al., 2004). These studies support a role of phasic dopamine release in the NAc core in encoding information about goal-directed behavior for natural rewards. The current study was designed to further characterize dopamine release events within the core and shell during cue-signaled goal-directed actions for a natural (sucrose) reward. Specifically, FSCV was used to monitor rapid dopamine release within each NAc subregion during a task in which an instructional cue was separated in time from the lever press response and sucrose delivery. The results show that although the sucrose predictive cue-evoked increases in phasic dopamine release in both the core and shell, sub-regional differences were observed in the magnitude of the cue-evoked response. Further, secondary dopamine release events were observed following the lever press response for sucrose in the NAc shell, but not the core.

\section{Methods}

\subsection{Sucrose self-administration}

Male Sprague-Dawley rats $(n=11)$ weighting $280-330 \mathrm{~g}$ were uses as subjects. Animals were individually housed with a 12-h light/12-h dark cycle and maintained at no less than $85 \%$ of pre-experimental body weight by food restriction (10-15 $\mathrm{g}$ of Purina laboratory chow each day, in addition to approximately $2.7 \mathrm{~g}$ of sucrose consumed during daily sessions). Food restriction was in place for the duration of behavioral testing except during the post-surgery recovery period, when food was given ad libitum. All procedures were performed in accordance with the University of North Carolina at Chapel Hill Institutional Animal Care and Use Committee.

Experimental sessions occurred in a $43 \times 43 \times 53 \mathrm{~cm}$ Plexiglas operant chamber that contained a retractable lever with a cue light above it. Rats were trained to self-administer sucrose pellets during single daily sessions ( 60 trials/session; 5 days/week). The beginning of the session was signaled by the onset of a house light and white noise. After a variable interval (initially VI 5-25 s, then gradually increased over $\sim 5-7$ days to VI 30-60 s) a tonelight cue was presented and $2 \mathrm{~s}$ later a lever extended into the chamber. A lever press (FR1 schedule of reinforcement) within $15 \mathrm{~s}$ after lever extension resulted in the delivery of a single sucrose pellet $(45 \mathrm{mg})$ into the receptacle, retraction of the lever, and termination of the audio-visual cue. Thus, only one sucrose reinforcer could be earned per trial. Failure to respond within $15 \mathrm{~s}$ after lever extension resulted in retraction of the lever, termination of 
the audiovisual cue and initiation of the variable interval (Fig. 1), and was considered an omission error.

\subsection{Surgical procedures}

Following the establishment of stable responding (characterized as no more than 2 omission errors per session for 3 consecutive sessions), animals were surgically prepared for voltammetric recordings. Rats were anesthetized with intramuscular injections of ketamine $(100 \mathrm{mg} / \mathrm{kg}$ ) and xylazine $(20 \mathrm{mg} / \mathrm{kg})$. A guide cannula (Bioanalytical Systems, West Lafayette, IN, USA) was stereotaxically positioned above the NAc core (1.3 $\mathrm{mm}$ anterior, $1.3 \mathrm{~mm}$ lateral relative to bregma) or shell $(1.7 \mathrm{~mm}$ anterior, $0.8 \mathrm{~mm}$ lateral relative to bregma) and a bipolar stimulating electrode (Plastics One, Roanoke, VA, USA) was ipsilaterally implanted in the VTA $(5.2 \mathrm{~mm}$ posterior, $1.0 \mathrm{~mm}$ relative to bregma, $7.5 \mathrm{~mm}$ ventral from dural surface). In addition, another guide cannula for the reference $\mathrm{Ag} / \mathrm{AgCl}$ electrode was placed in the contralateral hemisphere.

\subsection{Electrode fabrication}

Cylindrical carbon-fiber microelectrodes were prepared as described in detail previously (Cahill et al., 1996; Owesson-White et al., 2009). Briefly, a T650 carbon-fiber (6 $\mu \mathrm{m}$ diameter, Amoco Corporation Greenville, SC, USA) was inserted into a glass capillary (0.6 $\mathrm{mm}$ outer diameter, $0.4 \mathrm{~mm}$ inner diameter, A-M System, Carlsborg, WA, USA). The glass capillary was then pulled using a vertical puller (Narishige, Tokyo, Japan) and the exposed carbon-fiber was cut to $75-100 \mu \mathrm{m}$.

\subsection{Voltammetric recording}

After surgery, rats were allowed to recover to their pre-surgery body weight (at least 5 days of recovery). On the day of the experiment, a carbon-fiber microelectrode (50-100 $\mu \mathrm{m}$ of exposed fiber) was lowered into the NAc shell or core using a locally constructed microdrive (University of North Carolina at Chapel Hill, Department of Chemistry Instrument Shop), after placing an $\mathrm{Ag} / \mathrm{AgCl}$ reference electrode in the contralateral hemisphere. Wires from the reference and stimulating electrodes and a head-stage amplifier connected to the carbon-fiber electrode were routed to a swivel (Med. Associates, Inc.), which allowed free movement, and then to custom-built amplifiers (Chemistry Department Electronic Facility, University of North Carolina Chapel Hill, Chapel Hill, NC, USA) and into a computer interface. The carbon-fiber microelectrode was held at $-0.4 \mathrm{~V}$ versus $\mathrm{Ag} / \mathrm{AgCl}$ reference electrode and periodically a cyclic voltammogram was acquired ( $200 \mathrm{~ms}$ intervals) by applying a triangular waveform that drove the potential to $1.3 \mathrm{~V}$ and back to $-0.4 \mathrm{~V}$. Recordings were made at sites where electrical stimulation ( $125 \mu \mathrm{A}$ biphasic pulses, $2 \mathrm{~ms}$ per phase, $60 \mathrm{~Hz}, 24$ pulses) evoked detectable dopamine release prior to the start of the behavioral session (30 trials/session). Once confirmed, dopamine was measured during the behavioral task. In some animals $(n=6)$, the electrode was lowered $\sim 300 \mu \mathrm{m}$ until another release site was found after the completion of the experiment and dopamine release was measured during another behavioral session.

\subsection{Electrode calibration}

After recordings, carbon-fiber microelectrodes were calibrated in a solution of known dopamine concentration to convert measured changes in current to differential concentrations, as previously described (Longman et al., 2000; Phillips et al., 2003). Briefly, electrodes were placed in a flow-cell apparatus in a constant stream of buffer. Periodically the fluid was switched with buffer containing known concentrations of dopamine and the voltammetric signal measured was used to estimate the current/concentration ratio for dopamine. 


\subsection{Data analysis}

To confirm that dopamine was measured during the task, voltammetry data was analyzed using principal component analysis as previously described (Heien et al., 2005; Keithley et al., 2010). Once confirmed, changes in extracellular DA concentration during the task were assessed by aligning DA concentration traces to relevant behavioral events (cue presentations and lever press responses). To examine changes in dopamine relative to cue onset, a one-way repeated measures ANOVA followed by Tukey's HSD test was used to examined dopamine concentration during a baseline period ( $5 \mathrm{~s}$ before the cue onset) compared with dopamine concentration in successive $200 \mathrm{~ms}$ bins within a $2 \mathrm{~s}$ period after cue onset. To examine changes in dopamine relative to the lever press response, one-way repeated measures ANOVA followed by Tukey's HSD test examined dopamine concentration during the same baseline period ( $5 \mathrm{~s}$ before cue onset) compared with dopamine levels within successive $200 \mathrm{~ms}$ bins in a $5 \mathrm{~s}$ period after the lever press. Changes in dopamine concentration relative to cue onset or the sucrose-reinforced response were evaluated separately for core and shell. To directly compare dopamine release dynamics across the core versus shell following cue onset measurements were averaged into $1 \mathrm{~s}$ time bins. Changes in dopamine concentration were assessed using a two-way ANOVA with a main effect of region (core versus shell) as between-subject factors, and time (1 s time bins measurements) as repeated measures. If significant effects or interactions were obtained with an ANOVA, multiple pair-wise contrasts were conducted using Tukey's post-hoc test.

Unpaired $t$ tests were used to compare increases in extracellular dopamine in the core versus shell within $5 \mathrm{~s}$ after the lever press for sucrose versus baseline concentrations ( $5 \mathrm{~s}$ before cue onset). Linear regression analyses were completed that correlated signal-to-baseline $(\mathrm{S}: \mathrm{B})$ ratios for peak [DA] after cue onset versus peak $\mathrm{S}: \mathrm{B}$ ratio [DA] after the lever press for both NAc shell and core. Baseline was defined as the $10 \mathrm{~s}$ period prior to cue onset; signal was defined as [DA] $2 \mathrm{~s}$ after cue onset or the lever press response. S:B ratios were determined by dividing the signal by the baseline values.

All statistical analyses were carried out using GraphPad Prism 4.0 for Windows (GraphPad Software, La Jolla, CA) or Statistica for Windows (StatSoft, Tulsa, OK).

\subsection{Histology}

After completion of each experiment, rats were deeply anesthetized with a ketamine/ xylazine mixture $(100 \mathrm{mg} / \mathrm{kg}$ and $20 \mathrm{mg} / \mathrm{kg}$, respectively) and histological identification of electrode placement was completed using established procedures (Roitman et al., 2004; Day et al., 2007). Briefly, a stainless steel electrode housed in the same micromanipulator used during the experiment was lowered to the experimental recording site and a small electrolytic lesion was made $(50-500 \mu \mathrm{A}, 5 \mathrm{~s})$. Rats were then transcardially perfused with $300 \mathrm{~mL}$ of saline followed by $300 \mathrm{~mL}$ of $10 \%$ formalin. Each brain was removed and, after post fixing and freezing, sliced into $40 \mu \mathrm{m}$ coronal sections. Brain sections were mounted on slides, stained with thionin, coverslipped, and viewed with bright-field microscopy.

Histological reconstruction of electrode placements showing recording locations in the NAc core and shell are shown in Fig. 2.

\section{Results}

\subsection{Dopamine response to the reward predictive cue during sucrose self-administration}

All animals $(n=10)$ exhibited stable behavioral performance on the task on the recording day, with a mean latency to lever press following lever extension of $322 \pm 56 \mathrm{~ms}$. Fig. 3 shows a color representation of a set of background-subtracted cyclic voltammograms and the corresponding dopamine concentration trace in the NAc core and shell averaged across 
30 trials/session for a single animal. In the NAc core (left), the onset of the audio-visual cue (indicated by red dashed line at ' $C$ ') induced an increase in rapid dopamine release that reached a maximum of $47 \mathrm{nM}$ immediately after the presentation of the tone-light cue. The signal declined after cue onset, even following lever extension (at blue dashed line at ' $L$ ') and a response. In a representative recording in the NAc shell of another rat (right), cueevoked dopamine release reached a peak of $62 \mathrm{nM}$ after cue presentation. However, a second lower peak of $36 \mathrm{nM}$ was evident after lever extension followed by the sucrosereinforced response.

The average dopamine response measured in 7 locations across the NAc core of 4 well trained rats is shown in Fig. 4A. Cue-evoked increases in extracellular dopamine occurred immediately after cue onset (dashed black line) with a peak of $36 \pm 3 \mathrm{nM}$, and declined as the lever was extended into the chamber (dashed gray line). A one-way repeated measures ANOVA revealed a main effect of time $\left(F_{26,156}=30.95, p<0.0001\right)$. Tukey's post-hoc test revealed significant increases in extracellular dopamine immediately after cue onset $(p<$ 0.05 ) that declined at lever extension. Fig. 4B shows the average dopamine concentration measured in 10 locations across the NAc shell of 7 well trained animals. Cue-evoked dopamine release reached a peak of $54 \pm 8 \mathrm{nM}$ (dashed black line) immediately after the presentation of the audio-visual cue with a second peak of $14 \pm 3 \mathrm{nM}$ after the lever extension (indicated by gray dashed line). A one-way repeated measures ANOVA revealed a main effect of time $\left(F_{26,234}=23.49, p<0.0001\right)$. Tukey's post-hoc test showed significant increases in dopamine release immediately after the presentation of the audio-visual cue ( $p<$ $0.05)$; at $5 \mathrm{~s}$ after cue onset dopamine levels were still significantly higher than baseline.

\subsection{Increased dopamine relative to the sucrose-reinforced response in the NAc shell but not the core}

To determine if changes in rapid dopamine signaling occurred relative to the lever press response for sucrose, dopamine traces across sub-regions were aligned to the lever press response, shown in Fig. 5. In the NAc core (left), a one-way repeated measures ANOVA revealed a main effect of time $\left(F_{25,150}=3.45, p<0.0001\right)$. However, a Tukey's post-hoc test revealed that extracellular dopamine levels occurred before $(p<0.05)$, but not after the operant response (indicated by "R" and gray line) relative to baseline. In the NAc shell (right), a one-way ANOVA revealed a main effect of time $\left(F_{25,225}=10.38, p<0.0001\right)$. In contrast to the core however, Tukey's post-hoc test revealed significant changes in dopamine release $(p<0.05)$ immediately after the lever press response (indicated by " $\mathrm{R}$ " and dashed gray line) that declined to baseline levels $2.6 \mathrm{~s}$ after the response.

\subsection{Comparison of core and shell differences in rapid dopamine signaling during sucrose- directed behavior}

The temporal characteristics of extracellular dopamine release dynamics differ across the NAc shell and core. In the NAc shell, increases in phasic dopamine are larger and of longer duration to those in the core, relative to cue onset. Fig. 6 shows dopamine traces across the NAc shell and core where measurements were averaged into $1 \mathrm{~s}$ time bins across all sucrose self-administration sessions relative to cue onset (at time 0). A two-way ANOVA revealed a main effect of region $\left(F_{1,15}=8.18, p<0.05\right)$ and time $\left(F_{10,150}=37.22, p<0.0001\right)$ and a significant region $\times$ time interaction $\left(F_{10,150}=2.68, p<0.0001\right)$. Tukey's post-hoc test revealed a significant increase in extracellular dopamine over baseline in both the NAc core $(p<0.001)$ and shell $(p<0.05)$. However, significant increases in dopamine in the core lasted only $2 \mathrm{~s}$ after cue onset while increases in extracellular dopamine in the NAc shell were maintained until $6 \mathrm{~s}$ after the cue onset. Finally, the relationship between dopamine release evoked by the cue versus that observed immediately following the lever press response was examined using a linear regression analyses. Specifically, signal-to-baseline 
$(\mathrm{S}: \mathrm{B})$ ratios for peak dopamine concentration $[\mathrm{DA}]$ after cue onset were regressed relative to $S: B$ ratios for peak [DA] after the lever press (Fig. 7). For the NAc shell, a significant correlation was observed between S:B [DA] after cue onset and S:B [DA] after the lever press $\left(F_{1,8}=11.47, p<0.01, r^{2}=0.59\right)$. In contrast, for the NAc core, the correlation between these two events was not significant $\left(F_{1,5}=1.05, p=0.35, r^{2}=0.17\right)$.

\section{Discussion}

In previous studies, we reported differences in the development of phasic dopamine transmission patterns across the NAc shell and core during the acquisition of cue-drug associations (Aragona et al., 2009). We also showed that during instrumental responding for a natural (sucrose) reward, cues that signal sucrose availability contingent upon a response elicit increases in rapid dopamine signaling within the NAc core (Roitman et al., 2004; Jones et al., 2010b). Here, we extended those findings and investigated if differences in dopamine release dynamics occur within discrete NAc sub-regions during cue-signaled operant behavior for sucrose reward. The behavioral task used was designed to dissociate dopamine release during cue presentation and operant behavior. Our findings show that a sucrose predictive cue elicited increases in phasic dopamine release in both the shell and core subregions of the NAc. However, regional differences were observed in the magnitude and dynamics of those release events. Specifically, increases in extracellular dopamine were higher and longer lasting in the shell compared with the core. Moreover, a secondary dopamine release event occurred following the lever press response in the shell but not in the core.

Schultz and colleagues proposed that phasic changes in dopamine cell firing provide a 'prediction error' signal that compares expected outcomes with actual outcomes (Schultz et al., 1997). That is, unexpected rewards produce brief synchronous bursts among dopamine neurons, while fully predicted rewards typically evoke little or no phasic activity. Further, the events that serve as predictors (i.e., reward predictive cues) come to elicit brief bursts of dopaminergic neurons, and the magnitude of these conditioned neuronal responses is correlated with the certainty of the reward being predicted (Fiorillo et al., 2003). Dopamine concentration transients in terminal regions arise from phasic firing of dopaminergic neurons (Sombers et al., 2009). Thus, our results are those anticipated for the view that dopamine release in the NAc serves as a 'learning signal' since release events were reliably observed to reward predictive cues in both sub-regions. However, we find in the shell that cue-evoked release events are greater than in the core and that they also occur at the lever response. These differences are consistent with anatomical studies showing that dopaminergic projections from the VTA to the shell and the core are not uniform, but instead arise from two distinct groups of ventral midbrain neurons (Ikemoto, 2007). Thus, although both the NAc core and shell may play a key role in reward learning, the dynamics of that processing appear to be slightly different across NAc sub-regions. This finding implies differences in how dopamine may modulate NAc cell firing that encode reward cues and ultimately drive behavior.

Regional differences in dopamine release reported here are also supported by functional and anatomical studies showing heterogeneous distributions of dopamine (Voorn et al., 1989; Deutch and Cameron, 1992), dopamine uptake rates (Garris and Wightman, 1994) and dopamine uptake sites (Jones et al., 1996). The shell appears to have a greater tissue content of dopamine than the core (Voorn et al., 1989; Deutch and Cameron, 1992), but an average of half the number of dopamine uptake sites (Jones et al., 1996). The higher expression of dopamine transporters in the core correlates with neurochemical data showing that the NAc core exhibits faster clearance of dopamine with a $V_{\max }$ value about 3-fold higher than the shell (Garris and Wightman, 1994; Jones et al., 1996). Those anatomical distinctions might 
explain the more prolonged dopamine responses observed during the task in the shell. Such regional heterogeneity in dopamine fluctuations during operant conditioning for natural (sucrose) rewards supports the view that NAc shell and core encode different aspects of goal-directed actions.

In this regard, the NAc core has been implicated in the encoding of cue-reward learned associations (Day et al., 2007; Aragona et al., 2009) while the shell appears to be involved in primary reward processing associated with food and drugs (Pontieri et al., 1995, 1996; Tanda et al., 1997; Bassareo and Di Chiara, 1999; Aragona et al., 2008). For example, pharmacological inactivation of the NAc core, but not the shell, impairs cue-induced reinstatement for food and drug reward (Fuchs et al., 2004; Floresco et al., 2008). Moreover, studies employing lesions of the NAc show that only selective core lesions disrupt appetitive pavlovian approach behavior in an autoshaping procedure (Parkinson et al., 2000) and this effect appears to be dopamine dependent (Dalley et al., 2002). Conversely, the NAc shell, but not the core, appears to be implicated in processing hedonic and aversive responses to salient stimuli. For example, extracellular dopamine is increased by palatable and decreased by unpalatable taste stimuli selectively in the NAc shell (Wheeler et al., 2011). However the same study shows that dopamine release is increased by a cue that signals imminent cocaine delivery. Thus, it may be that cues that predict reward availability may or may not increase extracellular dopamine in the shell depending on the kind of conditioning employed (Pavlovian versus operant) and the subject's motivational state (Bassareo and Di Chiara, 1999; Cheng et al., 2003; Aragona et al., 2009; Owesson-White et al., 2009; Bassareo et al., 2011).

As such, differences in dopamine release dynamics relative to reward predictive cues appear to be dependent, in part, on the nature of the conditioning task as well as the type of reinforcer used. Using FSCV we previously reported that during pavlovian conditioning, cocaine-associated cues evoked increases in rapid dopamine signaling within the NAc core but not the shell (Aragona et al., 2009). However during cocaine self-administration increases in phasic dopamine release were observed in the NAc shell immediately before the lever press response for cocaine (Owesson-White et al., 2009). Interestingly, microdialysis studies indicate that during pavlovian conditioning tasks, a CS paired with food increases extracellular dopamine in the NAc core but not the shell (Bassareo and Di Chiara, 1999). Further, reports from the same laboratory show that non-contingent presentation of an instrumental CS resulted in a preferential DA response in the NAc shell (Bassareo et al., 2009, 2011). Collectively, these findings illustrate that reward predictive cues differentially alter dopamine release events within the NAc core and shell dependent upon a number of factors including the behavioral task and reinforcer type.

Further, dopamine changes in the NAc shell to cues that predict food availability appear to be dependent upon the animal's motivational state, a consequence of an increased incentive value of food by food deprivation. In fact, in non food-deprived rats, a food CS stimulated dopamine transmission in the NAc core (Bassareo and Di Chiara, 1999) but not in the shell, whereas in food-deprived rats a pavlovian CS increased extracellular dopamine in both shell and core sub-regions (Cheng et al., 2003). In the present study, where mildly food-deprived rats had to perform a cue-signaled instrumental task for sucrose reward, the instructional cue-evoked subsecond increases in dopamine release events in both core and shell. Collectively, these findings indicate that the animal's motivational state may play an important role in the encoding of food CSs in the NAc shell. That is, dopamine may play an important role when incentive salience is enhanced by deprivation or/and where the CS functions to instruct the animal to perform instrumental actions to obtain the reward. 
The present study also shows that dopamine release events remain elevated after cue presentation during the time of lever extension and relative to the lever press response for sucrose in the NAc shell but not the core. Further, in the shell, a significant correlation was observed between $\mathrm{S}: \mathrm{B}[\mathrm{DA}]$ after the cue onset and S:B [DA] after the lever press whereas no correlation was found for the NAc core. The positive correlation observed in the NAc shell indicates that the higher the cue-evoked dopamine release, the higher the dopamine release after the lever press response. One interpretation of this finding, consistent with the incentive salience hypothesis, is that dopamine release in the shell may function to attribute incentive salience to a reward predicting cue, potentiating a motivational state of wanting (Robinson and Berridge, 1993; Berridge and Robinson, 1998) that promotes goal-directed actions. In support, intra-shell infusions of amphetamine (a dopamine agonist) potentiates the ability of sucrose conditioned stimuli to increase instrumental responses for sucrose (Wyvell and Berridge, 2000) suggesting that the NAc shell may play an important role in using instructional cues to guide and modulate behavior (Saddoris et al., 2011).

The present study appears to be somewhat in contrast with a report by Wanat and colleagues (Wanat et al., 2010) in which no differences in dopamine release dynamics between shell and core were reported. In that study rats performed a task involving an FR4 or a progressive ratio schedule on alternative days to obtain food reward. Moreover, the onset of the reward predictive cue occurred at the same time as lever extension making it impossible to dissociate the effect of cue presentation and lever press responses on rapid dopamine signaling. Thus, the discrepancy between that report and the present findings may be due to differences in the behavioral task.

\section{Conclusions}

A number of studies have implicated a role of dopamine neurotransmission within the NAc shell in encoding primary aspects of drug and natural rewards (Di Chiara and Bassareo, 2007). In fact, addictive drugs and natural rewards share the ability of preferentially increasing extracellular dopamine within the NAc shell (Pontieri et al., 1995, 1996; Tanda et al., 1997; Bassareo and Di Chiara, 1999; Aragona et al., 2008). Here, we show that in the NAc shell, a second peak in rapid dopamine release is observed after the lever press response for sucrose, most likely during sucrose consumption, suggesting that such increases might mediate the primary reinforcing properties of sucrose. In contrast, within the NAc core, changes in extracellular dopamine were "time-locked" to the cue onset and further dopamine release events were not observed after the instrumental response for sucrose. In conclusion, the current study revealed that rapid dopamine signaling within the NAc core and shell is distinct and that each encodes specific aspects of goal-directed behaviors for "natural" (sucrose) reward.

\section{Acknowledgments}

We would like to thank Laura Ciompi for technical assistance and The University of North Carolina Department of Chemistry Electronics Facility for instrumentation. This research was supported by NIH, DA17318 to RMC.

\section{References}

Aragona BJ, Cleaveland NA, Stuber GD, Day JJ, Carelli RM, Wightman RM. Preferential enhancement of dopamine transmission within the nucleus accumbens shell by cocaine is attributable to a direct increase in phasic dopamine release events. J Neurosci. 2008; 28:8821-8831. [PubMed: 18753384]

Aragona BJ, Day JJ, Roitman MF, Cleaveland NA, Wightman RM, Carelli RM. Regional specificity in the real-time development of phasic dopamine transmission patterns during acquisition of a cuecocaine association in rats. Eur J Neurosci. 2009; 30:1889-1899. [PubMed: 19912327] 
Bassareo V, Di Chiara G. Differential influence of associative and non-associative learning mechanisms on the responsiveness of prefrontal and accumbal dopamine transmission to food stimuli in rats fed ad libitum. J Neurosci. 1997; 17:851-861. [PubMed: 8987806]

Bassareo V, Di Chiara G. Differential responsiveness of dopamine transmission to food-stimuli in nucleus accumbens shell/core compartments. Neuroscience. 1999; 89:637-641. [PubMed: 10199600]

Bassareo V, De Luca MA, Di Chiara G. Differential impact of pavlovian drug conditioned stimuli on in vivo dopamine transmission in the rat accumbens shell and core and in the prefrontal cortex. Psychopharmacology (Berl). 2007; 191:689-703. [PubMed: 17072592]

Bassareo, V.; Musio, P.; Lecca, D.; Di Chiara, G. Mesolimbic dopamine responsiveness to food conditioned stimuli after instrumental coniditioning paradigm. Behav Pharmacol; Abstracts of the 13th Biennal EBPS meeting; 2009. p. S37

Bassareo V, Musio P, Di Chiara G. Reciprocal responsiveness of nucleus accumbens shell and core dopamine to food- and drug-conditioned stimuli. Psychopharmacology (Berl). 2011; 214:687-697. [PubMed: 21110007]

Berridge KC, Robinson TE. What is the role of dopamine in reward: hedonic impact, reward learning, or incentive salience? Brain Res Rev. 1998; 28:309-369. [PubMed: 9858756]

Cacciapaglia F, Wightman RM, Carelli RM. Rapid dopamine signaling differentially modulates distinct microcircuits within the nucleus accumbens during sucrose-directed behavior. J Neurosci. 2011; 31:13860-13869. [PubMed: 21957248]

Cahill P, Walker QD, Finnegan JM, Mickelson GE, Wightman RM. Microelectrodes for the measurement of catecholamines in biological systems. Anal Chem. 1996; 68:3180-3186. [PubMed: 8797378]

Cheng JJ, de Bruin JP, Feenstra MG. Dopamine efflux in nucleus accumbens shell and core in response to appetitive classical conditioning. Eur J Neurosci. 2003; 18:1306-1314. [PubMed: 12956729]

Corbit LH, Muir JL, Balleine BW. The role of the nucleus accumbens in instrumental conditioning: evidence of a functional dissociation between accumbens core and shell. J Neurosci. 2001; 21:3251-3260. [PubMed: 11312310]

Dalley JW, Chudasama Y, Theobald DE, Pettifer CL, Fletcher CM, Robbins TW. Nucleus accumbens dopamine and discriminated approach learning: interactive effects of 6-hydroxydopamine lesions and systemic apomorphine administration. Psychopharmacology (Berl). 2002; 161:425-433. [PubMed: 12073171]

Day JJ, Roitman MF, Wightman RM, Carelli RM. Associative learning mediates dynamic shifts in dopamine signaling in the nucleus accumbens. Nat Neurosci. 2007; 10:1020-1028. [PubMed: 17603481]

Deutch AY, Cameron DS. Pharmacological characterization of dopamine systems in the nucleus accumbens core and shell. Neuroscience. 1992; 46:49-56. [PubMed: 1350665]

Di Chiara G, Bassareo V. Reward system and addiction: what dopamine does and doesn't do. Curr Opin Pharmacol. 2007; 7:69-76. [PubMed: 17174602]

Fiorillo CD, Tobler PN, Schultz W. Discrete coding of reward probability and uncertainty by dopamine neurons. Science. 2003; 299:1898-1902. [PubMed: 12649484]

Floresco SB, McLaughlin RJ, Haluk DM. Opposing roles for the nucleus accumbens core and shell in cue-induced reinstatement of food-seeking behavior. Neuroscience. 2008; 154:877-884. [PubMed: 18479836]

Fuchs RA, Evans KA, Parker MC, See RE. Differential involvement of the core and shell subregions of the nucleus accumbens in conditioned cue-induced reinstatement of cocaine seeking in rats. Psychopharmacology (Berl). 2004; 176:459-465. [PubMed: 15138757]

Garris PA, Wightman RM. Different kinetics govern dopaminergic transmission in the amygdala, prefrontal cortex, and striatum: an in vivo voltammetric study. J Neurosci. 1994; 14:442-450. [PubMed: 8283249]

Heien ML, Khan AS, Ariansen JL, Cheer JF, Phillips PE, Wassum KM, Wightman RM. Real-time measurement of dopamine fluctuations after cocaine in the brain of behaving rats. Proc Natl Acad Sci U S A. 2005; 102:10023-10028. [PubMed: 16006505] 
Heimer L, Zahm DS, Churchill L, Kalivas PW, Wohltmann C. Specificity in the projection patterns of accumbal core and shell in the rat. Neuroscience. 1991; 41:89-125. [PubMed: 2057066]

Ikemoto S. Dopamine reward circuitry: two projection systems from the ventral midbrain to the nucleus accumbens-olfactory tubercle complex. Brain Res Rev. 2007; 56:27-68. [PubMed: 17574681]

Jones SR, O'Dell SJ, Marshall JF, Wightman RM. Functional and anatomical evidence for different dopamine dynamics in the core and shell of the nucleus accumbens in slices of rat brain. Synapse. 1996; 23:224-231. [PubMed: 8807751]

Jones JL, Day JJ, Wheeler RA, Carelli RM. The basolateral amygdala differentially regulates conditioned neural responses within the nucleus accumbens core and shell. Neuroscience. 2010a; 169:1186-1198. [PubMed: 20570714]

Jones JL, Day JJ, Aragona BJ, Wheeler RA, Wightman RM, Carelli RM. Basolateral amygdala modulates terminal dopamine release in the nucleus accumbens and conditioned responding. Biol Psychiatry. 2010b; 67:737-744. [PubMed: 20044074]

Jongen-Relo AL, Voorn P, Groenewegen HJ. Immunohistochemical characterization of the shell and core territories of the nucleus accumbens in the rat. Eur J Neurosci. 1994; 6:1255-1264. [PubMed: 7526940]

Keithley RB, Carelli RM, Wightman RM. Rank estimation and the multivariate analysis of in vivo fast-scan cyclic voltammetric data. Anal Chem. 2010; 82:5541-5551. [PubMed: 20527815]

Longman MJ, Budygin EA, Gainetodinov RR, Wightman RM. Quantitation of in vivo measurements with carbon fiber microelectrodes. J Neurosci Methods. 2000; 95:95-102. [PubMed: 10752479]

Owesson-White CA, Ariansen J, Stuber GD, Cleaveland NA, Cheer JF, Wightman RM, Carelli RM. Neural encoding of cocaine-seeking behavior is coincident with phasic dopamine release in the accumbens core and shell. Eur J Neurosci. 2009; 30:1117-1127. [PubMed: 19735286]

Parkinson JA, Willoughby PJ, Robbins TW, Everitt BJ. Disconnection of the anterior cingulate cortex and nucleus accumbens core impairs Pavlovian approach behavior: further evidence for limbic cortical-ventral striatopallidal systems. Behav Neurosci. 2000; 114:42-63. [PubMed: 10718261]

Paxinos, G.; Watson, C. The Rat Brain in Stereotaxic Coordinates. Elsevier Academic Press; New York, NY: 2005.

Phillips PE, Robinson DL, Stuber GD, Carelli RM, Wightman RM. Real tome measurements of phasic changes in extracellular dopamine concentration in freely moving rats by fast-scan cyclic voltammetry. Methods Mol Med. 2003; 79:443-464. [PubMed: 12506716]

Pontieri FE, Tanda G, Di Chiara G. Intravenous cocaine, morphine, and amphetamine preferentially increase extracellular dopamine in the "shell" as compared with the "core" of the rat nucleus accumbens. Proc Natl Acad Sci U S A. 1995; 92:12304-12308. [PubMed: 8618890]

Pontieri FE, Tanda G, Orzi F, Di Chiara G. Effects of nicotine on the nucleus accumbens and similarity to those of addictive drugs. Nature. 1996; 382:255-257. [PubMed: 8717040]

Robinson TE, Berridge KC. The neural basis of drug craving: an incentive-sensitization theory of addiction. Brain Res Rev. 1993; 18:247-291. [PubMed: 8401595]

Roitman MF, Stuber GD, Phillips PE, Wightman RM, Carelli RM. Dopamine operates as a subsecond modulator of food seeking. J Neurosci. 2004; 24:1265-1271. [PubMed: 14960596]

Saddoris MP, Stamatakis A, Carelli RM. Neural correlates of Pavlovian-to-instrumental transfer in the nucleus accumbens shell are selectively potentiated following cocaine self-administration. Eur $\mathbf{J}$ Neurosci. 2011

Schultz W, Dayan P, Montague PR. A neural substrate of prediction and reward. Science. 1997; 275:1593-1599. [PubMed: 9054347]

Sombers LA, Beyene M, Carelli RM, Wightman RM. Synaptic overflow of dopamine in the nucleus accumbens arises from neuronal activity in the ventral tegmental area. J Neurosci. 2009; 29:17351742. [PubMed: 19211880]

Tanda G, Pontieri FE, Di Chiara G. Cannabinoid and heroin activation of mesolimbic dopamine transmission by a common mu1 opioid receptor mechanism. Science. 1997; 276:2048-2050. [PubMed: 9197269] 
Voorn P, Gerfen CR, Groenewegen HJ. Compartmental organization of the ventral striatum of the rat: immunohistochemical distribution of enkephalin, substance $\mathrm{P}$, dopamine, and calcium-binding protein. J Comp Neurol. 1989; 289:189-201. [PubMed: 2478598]

Wanat MJ, Kuhnen CM, Phillips PE. Delays conferred by escalating costs modualte dopamine release to rewards but not their predictors. J Neurosci. 2010; 30:12020-12027. [PubMed: 20826665]

Wheeler RA, Aragona BJ, Fuhrmann KA, Jones JL, Day JJ, Cacciapaglia F, Wightman RM, Carelli RM. Cocaine cues drive opposing context-dependent shifts in reward processing and emotional state. Biol Psychiatry. 2011; 69:1067-1074. [PubMed: 21481843]

Wyvell CL, Berridge KC. Intra-accumbens amphetamine increases the conditioned incentive salience of sucrose reward: enhancement of reward "wanting" without enhanced "liking" or response reinforcement. J Neurosci. 2000; 20:8122-8130. [PubMed: 11050134]

Zahm DS, Brog JS. On the significance of subterritories in the "accumbens" part of the rat ventral striatum. Neuroscience. 1992; 50:751-767. [PubMed: 1448200]

Zahm DS, Heimer L. Specificity in the efferent projections of the nucleus accumbens in the rat: comparison of the rostral pole projection patterns with those of the core and shell. J Comp Neurol. 1993; 327:220-232. [PubMed: 8425943] 


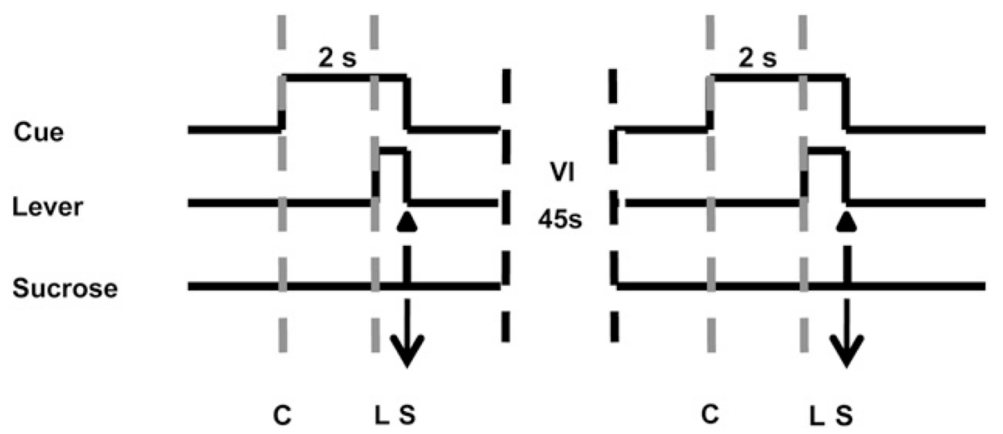

Fig. 1.

Schematic diagram of the behavioral task. Animals were presented with an audio-visual cue (indicated by "C") on a variable interval $45 \mathrm{~s}$ schedule (30-60 s). Two seconds later a lever extended into the chamber (indicated by "L"). Each response on the lever (denoted by arrow) resulted in delivery of a sucrose pellet (indicated by ' $S$ '), retraction of the lever and offset of the audio-visual cue. Failure to lever press within $15 \mathrm{~s}$ of lever extension resulted in retraction of the lever, termination of the audiovisual cue, and no sucrose delivery. Area between gray dashed lines denotes period between cue onset and lever extension. 


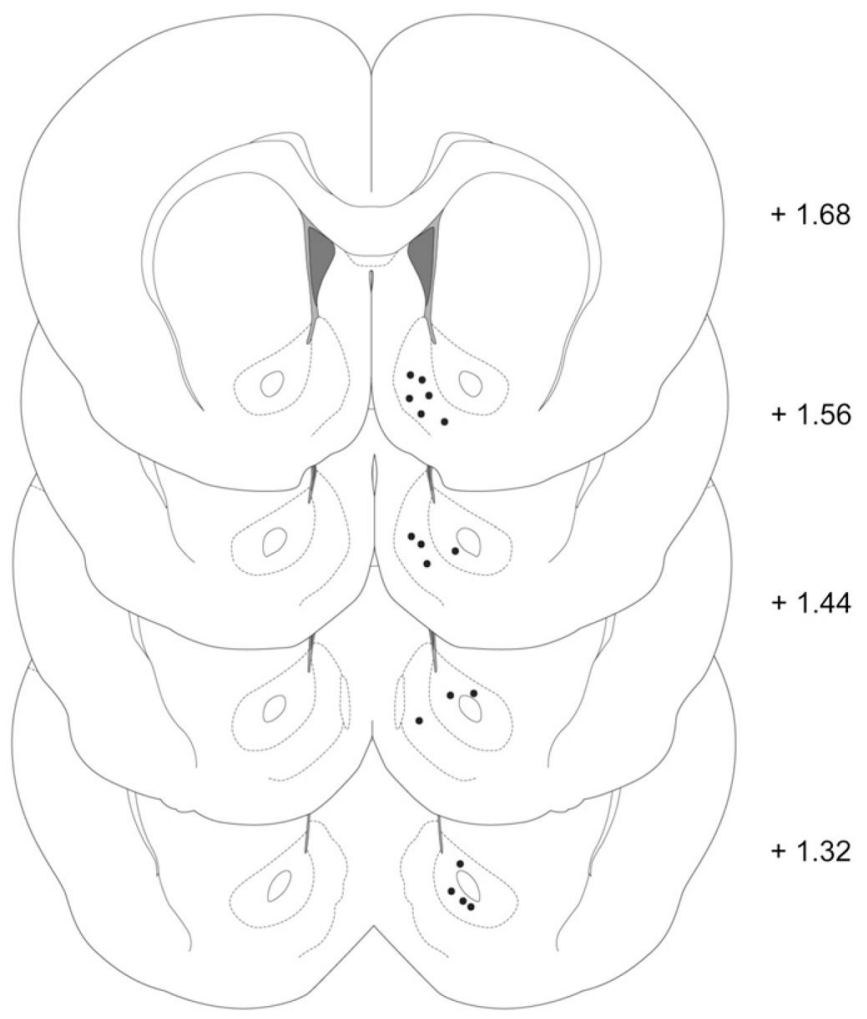

Fig. 2.

Coronal diagram illustrating confirmed locations of carbon-fiber microelectrodes tips in the NAc shell and core. Numbers to the right indicate the anteroposterior coordinates $( \pm 0.2 \mathrm{~mm})$ relative to bregma. Coordinates and drawings were taken from a stereotaxic atlas (Paxinos and Watson, 2005). 
A Core
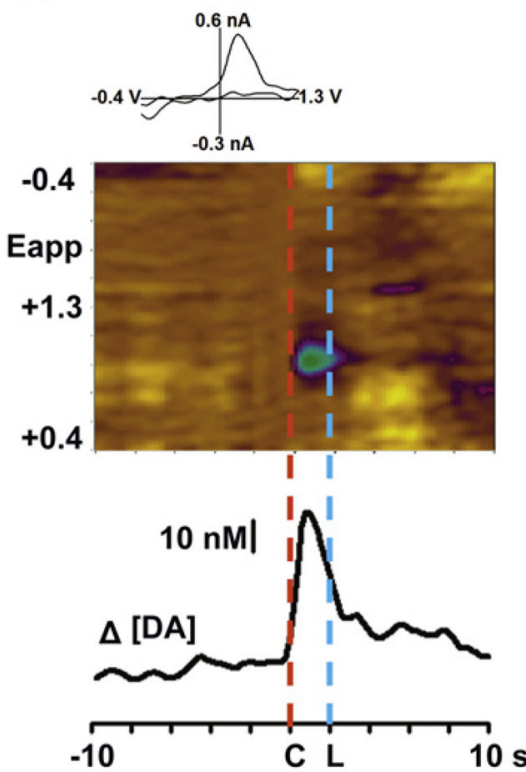

B Shell
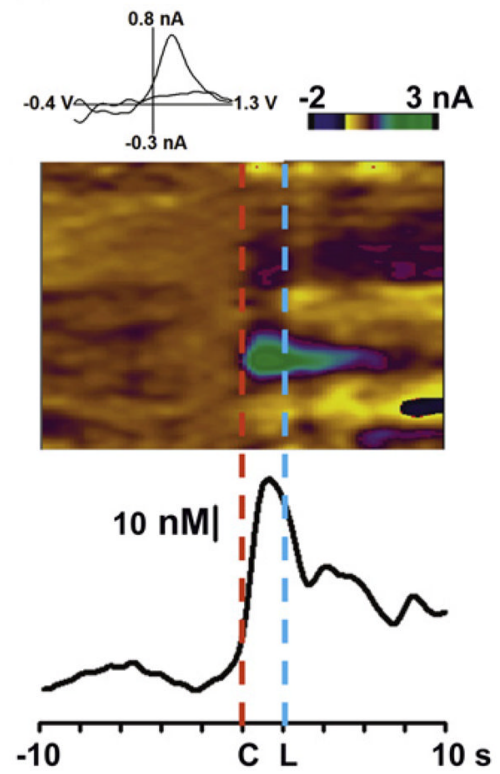

Fig. 3.

Changes in rapid dopamine release during sucrose self-administration for single animals averaged across a 30 trial session. The color plot (top) represents changes in current (color) by applied potential (Eapp, $y$-axis) over a 20-s time window ( $x$-axes) during the task in the NAc core (A) and shell (B). Fluctuations in extracellular dopamine are shown in the trace below, aligned in time to the color plot. Data are aligned to the cue onset (indicated by "C" at time 0); "L" indicates lever extension. 


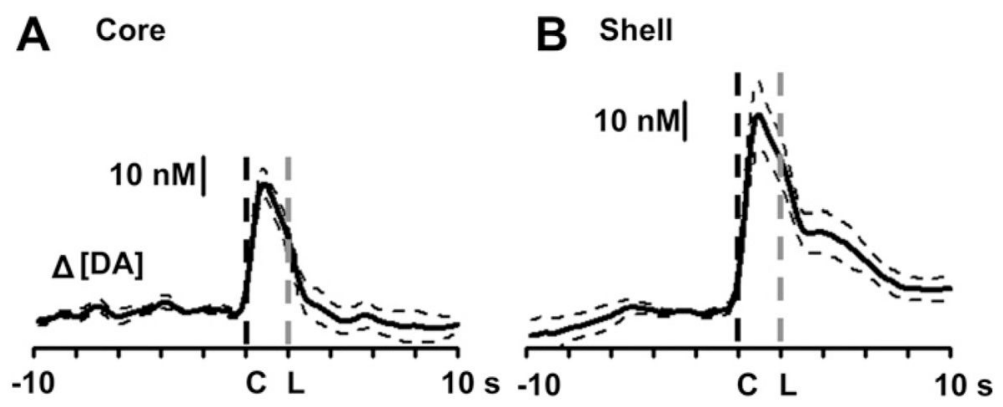

Fig. 4.

Average changes in NAc extracellular dopamine across all recording locations relative to cue onset (indicated by " $\mathrm{C}$ " at time 0$)$ for the NAc core $(\mathrm{A}, n=7)$ and shell $(\mathrm{B}, n=10)$. "L" is lever extension. Dashed traces represent standard error of the mean. 


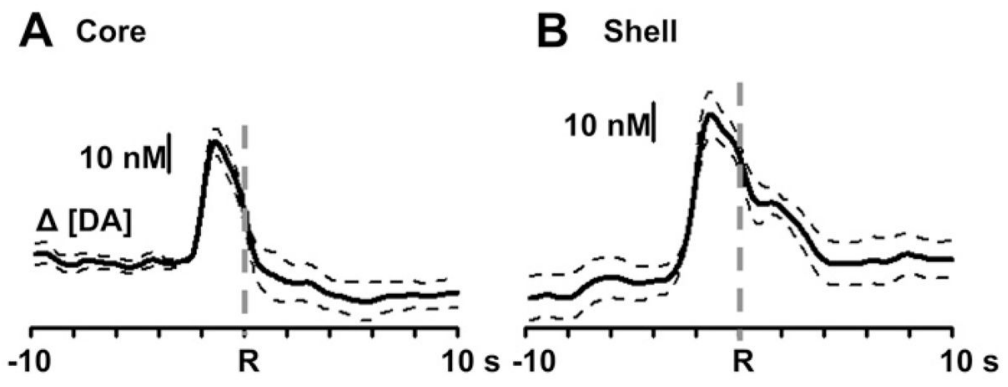

Fig. 5.

Average changes in dopamine release relative to the lever press response for sucrose across all recording locations. Data are aligned to the lever press response (indicated by " $R$ ") for the NAc core $(\mathrm{A}, n=7)$ and shell $(\mathrm{B}, n=10)$. Dashed traces represent standard error of the mean. 


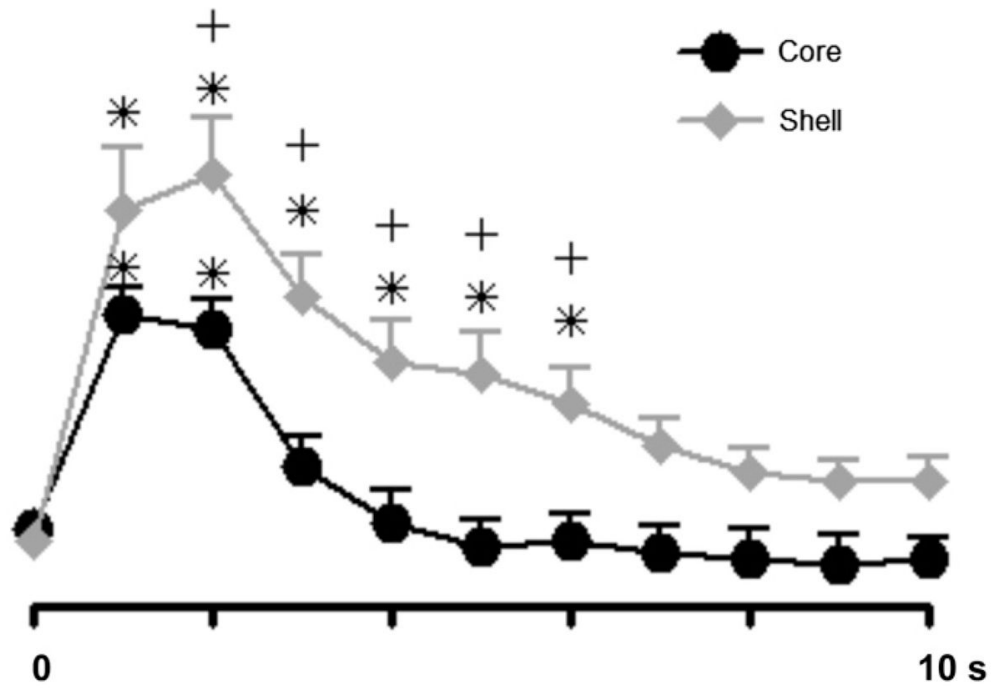

Fig. 6.

Comparison of changes in dopamine signaling across all recordings in the core and shell during sucrose self-administration. Dopamine concentration was separated into $1 \mathrm{~s}$ bins for comparison between core and shell during the task. Time 0 is cue onset. All data are mean \pm SEM. $* p<0.05$ versus baseline $;+p<0.05$ core versus shell. 

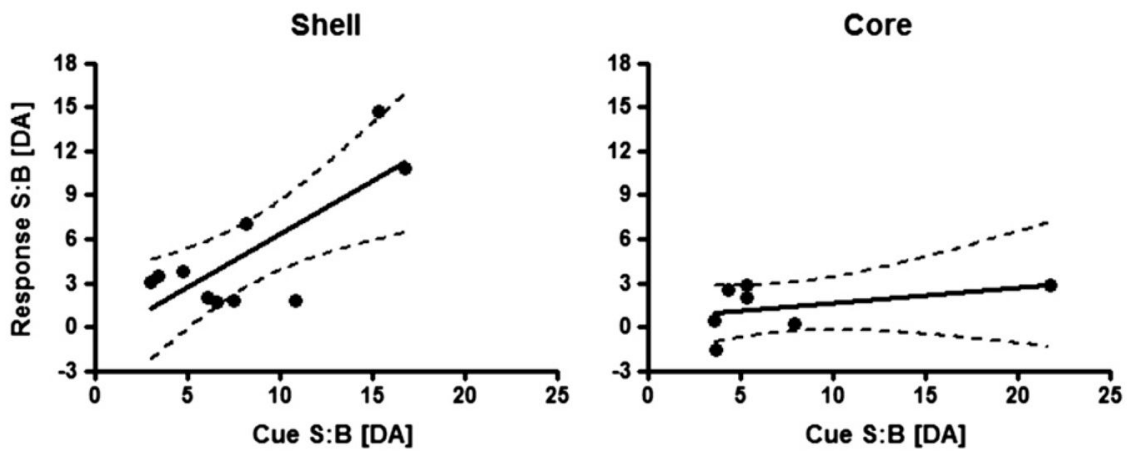

Fig. 7.

Linear regression analyses correlating $\mathrm{S}$ :B ratios for peak [DA] after cue onset versus $\mathrm{S}$ :B ratio for peak [DA] after the lever press for both NAc shell and core. For the NAc shell (left), a significant positive linear regression was observed. For the NAc core (right), the correlation was not significant. 